\title{
Significados e interpretaciones del trabajo infantil en contexto rural y urbano
}

\author{
Jenny Lisseth Avendaño-López, Ph. D. a \\ Universidad Surcolombiana, Colombia \\ Maribel Castillo-Caicedo, Ph. D. ${ }^{\text {b }}$ \\ Pontificia Universidad Javeriana, Colombia
}

jenny.avendano@usco.edu.co

\section{Resumen (analítico)}

El artículo propicia la comprensión del problema del trabajo infantil rural y urbano desde perspectiva interpretativa, a partir de los significados y las conceptualizaciones de las acciones de la práctica del trabajo, propias de niños y niñas en una zona rural del municipio de Tello y en la ciudad de Neiva en el departamento del Huila, Colombia. El enfoque metodológico empleado es una aproximación a la teoría fundamentada, configurada a partir de tres substanciales categorías: el capital social, el reconocimiento y el enfoque de capacidades. Se producen hallazgos respecto a la diferenciación sobre la lectura que los niños y niñas construyen sobre el significado de trabajo infantil remunerado, doméstico y relacionado con la dinámica y los vínculos que se tejen en el hogar y el entorno escuela.

\section{Palabras clave}

Capital social, reconocimiento, capacidades, trabajo infantil.

\section{Thesauro}

Tesauro de Ciencias Sociales de la Unesco.

\section{Para citar este artículo}

Avendaño-López, J. L., \& Castillo-Caicedo, M. (2021). Significados e interpretaciones del trabajo infantil en contexto rural y urbano. Revista Latinoamericana de Ciencias Sociales, Niñez y Juventud, 19(3), 1-19.

https://dx.doi.org/10.11600/rlcsnj.19.3.5077

\section{Historial}

Recibido: 03.05.2021

Aceptado: 28.07.2021

Publicado: 31.08.2021

\section{Información artículo}

Este artículo presenta resultados de la investigación titulada Factores de riesgo asociados a la vinculación de niños y niñas al trabajo infantil en Colombia. Esta investigación inició el primer semestre de 2016 y concluyó en marzo de 2021. Área: ciencias sociales; subárea: sociología. 


\section{Meanings and interpretations of child labor in rural and urban contexts}

\section{Abstract (analytical)}

The paper seeks to understand the problem of rural and urban child labor using an interpretive perspective based on the meanings and conceptualizations of child labour in in the rural municipality of Tello and the city of Neiva, both located in the department of Huila, Colombia. The methodological approach used is an approximation of grounded theory that is configured with three categories: social capital; recognition; and the capabilities approach. The authors identify differentiations in the meanings constructed by children regarding the meaning of paid child labor, domestic work and the dynamics and links that form part of their home and school environments.

\section{Keywords}

Social capital, recognition, skills, child labor.

\section{Significados e interpretações do trabalho infantil em contextos rurais e urbanos}

\section{Resumo (analítico)}

Este artigo promove a compreensão da problemática do trabalho infantil rural e urbano em uma perspectiva interpretativa, a partir dos significados e conceituações das ações da prática laboral, típicas de meninos e meninas de uma zona rural do município de Tello e na cidade de Neiva no departamento de Huila, Colômbia. A abordagem metodológica empregada é uma aproximação à teoria fundamentada, configurada a partir de três categorias substanciais: capital social, reconhecimento e abordagem das capacidades. São produzidos resultados sobre a diferenciação na leitura que meninos e meninas constroem sobre o significado de trabalho infantil remunerado, trabalho doméstico e em relação às dinâmicas e vínculos que se tecem no ambiente doméstico e escolar.

\section{Palavras-chave}

Capital social, reconhecimento, habilidades, trabalho infantil.

Información autoras

[a] Doctora en Ciencias Sociales. Niñez y Juventud, Universidad de Manizales-Cinde. Profesora Asociada Universidad Surcolombiana, Neiva, Colombia. (iD) 0000-0002-9663-6139. H5: 2. Correo electrónico: jenny.avendano@usco.edu.co

[b] Doctora en Ciencias Sociales, Niñez y Juventud, Universidad de Manizales-Cinde. Profesora Asociada Pontificia Universidad Javeriana Cali. iD 0000-0002-8578-9498. H5: 9. Correo electrónico: 


\section{Introducción}

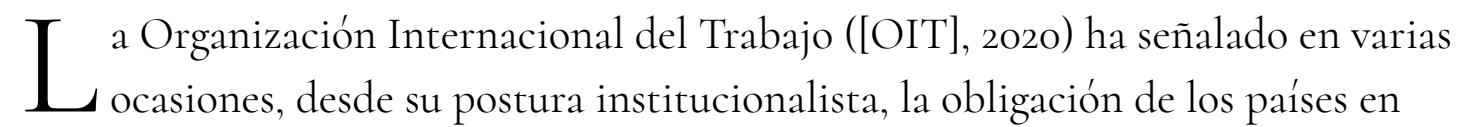
asumir una propuesta de política social que enfrente de manera contundente el trabajo infantil en condiciones estructurales y coyunturales.

Entre las causas que lo generan se documenta, entre otras, como una de las principales, a la pobreza y los factores asociados a ella (Carrión et al., 2021), como el ingreso familiar y la tasa de dependencia (Salazar, 200o), la falta de oportunidades laborales para los adultos del hogar del niño o la niña que trabaja y la inequitativa distribución de la riqueza en virtud de la reproducción social (Reséndez et al., 2019). Sin embargo, aunque la pobreza tiene una fuerte relación con el fenómeno, no se puede considerar este como el único determinante, pues se ha comprobado que la estructura del hogar puede incidir de manera importante en la incidencia del trabajo de los niños y las niñas (Simón et al., 2020). La participación de los niños y las niñas en el trabajo se inscribe en las dinámicas propias del territorio, lo cual permite extender un punto focal de análisis menos reduccionista y que promueve una comprensión del ambiente laboral infantil asociado a las realidades socioculturales propias de los niños y niñas (Rausky, 2020).

En este sentido, el propósito de este artículo es ofrecer un campo comprensivo sobre las construcciones conceptuales que niños y niñas en su ambiente natural de trabajo (rural y urbano) vivencian y que representa el modo como esa realidad es comprendida, interpretada y expresada por ellos y ellas (Bonilla \& Rodríguez, 200o; Lefebvre, 1982).

Este proceso de investigación cualitativa permitió validar la interpretación de la experiencia del niño y la niña que trabaja en su entorno familiar, social y económico, con lo cual se logró ampliar el análisis, en el marco del desarrollo de la tesis doctoral, de análisis mixto, sobre los factores que se asocian a la vinculación de niños y niñas al trabajo en Colombia. 
En virtud de posibilitar un análisis más amplio sobre el problema del trabajo infantil es necesario vincular aspectos relacionados con las construcciones sociales que se pueden producir desde los espacios naturales, en los que se vivencia la práctica del trabajo infantil. Autores como Frasco-Zuker (2016), García (2015) y Rausky (2020) consideran relevante la voz de las infancias trabajadoras desde una mirada contextual y reconociendo la visión de niños y niñas como sujetos con derechos y obligaciones, quienes cuentan con opinión en los asuntos que les atañe (Becerra \& Cayeros, 2017)

La investigación desarrollada dio lugar a responder la pregunta: ¿cuáles son los significados y conceptualizaciones en la construcción de las acciones que implica la práctica de trabajo infantil rural y urbano? Planteamiento que permitió diseñar una valoración sobre el desarrollo natural y espontáneo de los sucesos en torno a las prácticas de trabajo infantil rural y urbano.

La metodología empleada en el desarrollo de esta investigación es una aproximación a la teoría fundamentada, enfoque interpretativo, situado en la comprensión del significado de las acciones de los principales actores, de tal manera que tanto la interpretación de las realidades de los participantes en la investigación como la del investigador convergen para el análisis (Hernández-Sampieri et al., 2010). Es preciso advertir sobre el proceso previo de análisis cuantitativo en el marco de la investigación que este permitió orientar los ejes de análisis categóricos seleccionados en el proceso cualitativo, que para este caso son el capital social, el reconocimiento y las capacidades.

La relevancia del conocimiento y la comprensión de la realidad de los niños y las niñas trabajadores en contextos situados se respalda en posturas teóricas que entienden la realidad como un fenómeno que se construye socialmente (Berger \& Luckman, 2003).

Esta construcción de la realidad de los niños y niñas trabajadoras en el Municipio de Tello y en el microcentro de la ciudad de Neiva (departamento del Huila, Colombia) se estructura sistematizando, codificando y analizando los datos cualitativos recopilados mediante entrevistas y observación directa. A partir de este proceso, se busca comprender la realidad que han construido los participantes sobre el fenómeno del trabajo infantil en sus propios territorios. El proceso de análisis también tiene como propósito converger en ciertas categorías emergentes relacionadas con las propiedades y las características propias de las dinámicas del trabajo infantil para este grupo de participantes. 


\section{Precisiones sobre las categorías conceptuales}

En esta sección se exponen los aspectos teóricos relevantes que corresponden a las definiciones de trabajo infantil y de niñez; se conceptualiza el andamiaje teórico de los ejes de análisis categoriales definidos sobre capital social, reconocimiento y capacidades y que permitió articular el proceso metodológico según su operatividad y su posterior análisis.

\section{Conceptos de base para trabajo infantil y niñez}

Por definición de la OIT (2002) la expresión trabajo infantil suele definirse como «todo trabajo que priva a los niños de su niñez, su potencial y su dignidad, y que es perjudicial para su desarrollo físico y psicológico» (p. 12). Sin embargo, esta organización reconoce que no todos los trabajos son peligrosos si no afectan su salud física, emocional y si no interfieran con su proceso de escolarización. El trabajo infantil se refiere a actividades que representen peligro o que interfieran en su escolarización de manera parcial o total y desarrollado como la actividad económica que incluye todos los tipos de producción para el mercado de trabajo remunerado y ciertos tipos de trabajo que se realizan en casa considerados como labores domésticas (OIT, 2002).

Con respecto al concepto de niñez, la Convención sobre los Derechos del Niño, en la cual se ratifican todos los países de la subregión andina y el cono sur (Martínez, 2020), se reafirma en la obligación de facilitar a los niños y las niñas el cuidado y la asistencia necesarias en razón de su vulnerabilidad. Aquí resalta el papel protagónico de la familia en lo que respecta a la protección y la asistencia, así como la protección jurídica y no jurídica antes y después del nacimiento; adicionalmente, recalca el papel de la comunidad y los valores culturales en el desarrollo de la niñez (Unicef, 2006). En esta investigación se entiende que la norma de referencia para Colombia, como estado firmante, respecto al trabajo infantil es el artículo primero de dicha Convención: «Para los efectos de la presente Convención, se entiende por niño todo ser humano menor de dieciocho años de edad, salvo que, en virtud de la ley que le sea aplicable, haya alcanzado antes la mayoría de edad» (Unicef, 2006, p. 10).

\section{El trabajo infantil: una construcción teórica multidisciplinar}

Ahora bien, con respecto a la construcción teórica del trabajo infantil, es relevante para esta investigación tomar los referentes que desde distintas disciplinas aportan a su conceptualización y exploración. Son diversos los enfoques de análisis desde el campo del 
conocimiento, sin embargo, desde disciplinas como la economía, la sociología, la antropología y la psicología toma fuerza el discurso de producir un análisis multicausal que deriva en las prácticas de trabajo infantil. Autores como Bar y Basu (2009) y Basu (1998) exponen la importancia de dar sentido al análisis económico de las causas de trabajo infantil en un escenario contextualizado, a tal punto que puede ser posible la existencia de equilibrios múltiples, en donde logre ser eficiente social y económicamente la existencia del trabajo infantil. La problematización de la niñez desde las investigaciones antropológicas han direccionado el enfoque hacia la importancia de los significados y de las prácticas conexas a las etapas de la vida y cómo estas se reacondicionan según los contextos históricos, culturales y sociales incorporando en el análisis a la niñez (Frasco-Zuker, 2016). Estudios derivados del análisis sociológico sobre el trabajo infantil asumen teórica y epistemológicamente el estudio sobre el agenciamiento activo de los niños y niñas, capaces de construir lo social y dispuestos a reflexionar sobre sus vivencias (Rausky, 2020). Los desafíos en el abordaje teórico del trabajo infantil expuestos por Rausky se sitúan en deslocalizarse de la mirada y del análisis reduccionista que asocian al trabajo infantil a un mal en sí mismo y potencian la idea de analizar otros aspectos que constituyen el problema y que posibilitan revelar particularidades menos evidentes - pero igualmente relevantes- sobre el trabajo infantil.

En este mismo campo de estudio, avances expuestos por Zelizer (2002) resaltan la importancia sobre la observación directa en la participación de niños y niñas en actividades de producción, distribución y consumo, encontrando que de ellos y ellas sí existe una participación extensa y consecuente en la economía. La organización, los significados y las consecuencias de la actividad económica para las infancias trabajadoras, puede variar de manera importante en tres conjuntos diferentes de relaciones sociales: con otros miembros del hogar, con agentes fuera del hogar y con sus pares.

Este norte teórico propicia el ambiente de análisis argumentado en un contexto definido que, para este caso, es el trabajo infantil rural en una zona de producción cafetera (Municipio de Tello) y urbano en el microcentro de la ciudad capital en el departamento del Huila y en donde se explora la construcción conceptual y de significados de los niños y niñas a partir de sus experiencias de trabajo en casa y fuera de ella. 


\section{El capital social, el reconocimiento y las capacidades} como categorías de análisis para el trabajo infantil situado en contextos urbanos y rurales ${ }^{1}$

\section{Capital social}

Para aproximarse al capital social es preciso acudir a Pierre Bourdieu (1986) quien se refirió por primera vez a este término; con ello rompe con la concepción tradicional de capital, porque encuentra que en la producción de mercancías hay distintas expresiones del capital, y la capacidad de asociarse para producir conforma un capital diferente al económico y al cultural. En esta forma, para estudiar la vida social es indispensable comprender que el conocimiento sobre las acciones de los otros se inscribe en el campo de la subjetividad y esas miradas de los hechos particulares se relacionan con ámbitos basados en las posibilidades o probabilidades (Trujillo \& Álvarez, 2011).

El concepto de capital social estudiado por Coleman (1988) aporta al análisis de los problemas sociales y económicos; una definición teórica que permite vincular a la comprensión de las decisiones de los actores y los agentes sociales y económicos, otros elementos que se constituyen en el capital social. Entonces, es imprescindible comprender el curso de construcción de esta categoría de análisis que emerge de la estructura microsocial (familias nucleares, individuos) y la estructura macrosocial, con amplio alcance territorial y que impregna las estructuras sociales intermedias a través de, por ejemplo, normas que permiten a las personas interactuar en entornos de seguridad y que producen la confianza ciudadana.

\section{Reconocimiento situado}

Para Fraser (1997) es preciso vincular las dimensiones segregadas del reconocimiento y la redistribución, pues el esfuerzo por integrarlas puede conducir a ubicar un marco teórico adecuado que permita explicar las problemáticas sociales actuales. Las exigencias del cambio del paradigma económico que dé respuesta al porqué de los fenómenos sociales y al cómo solucionarlos, produce exigencias del cambio cultural, que se mezclan con

\footnotetext{
${ }^{1}$ La elección de estas familias categoriales tiene la intención de ser el anclaje con el desarrollo y resultado del proceso cuantitativo que antecedió el abordaje sobre la exploración de las vivencias y las prácticas de trabajo infantil en zona rural y urbana en el departamento del Huila y cuyos resultados se exponen en este manuscrito. La definición de estas emerge de los resultados de la modelación econométrica, la cual se definió en su estructura en tres focos de análisis: lo individual (reconocimiento), lo familiar (capital social) y educación y formación (capacidades). Los instrumentos construidos y el análisis de la información de los datos cualitativos se estructuran sobre este conjunto de categorías.
} 
los requerimientos del cambio económico y la injusticia socioeconómica que puede incluir la explotación o la marginación económica. Este tipo de injusticia se relaciona con la imposibilidad de acceder a oportunidades de ingresos en condiciones justas en términos de salario, ocupación, seguridad y dignidad.

Fraser (1997) resalta igualmente la injusticia simbólica, que «se naturaliza en los patrones sociales de representación, interpretación y comunicación» (p. 4). En este caso, el no reconocimiento de prácticas sociales asociadas al trabajo doméstico, que no se remunera porque culturalmente se vincula a la ayuda que las mujeres realizan en los hogares. Las labores realizadas en el hogar rural, en donde participan principalmente los padres junto con los hijos, y cómo estas otras situaciones en donde no media el proceso transaccional que vincule elementos como salario-contrato. La medición económica no logra enlazar este tipo de actividades al cuantificar la participación de la población económicamente activa, dado que no reconoce estas prácticas sociales como actividades de trabajo.

\section{El enfoque de las capacidades}

El desplazamiento teórico y conceptual que Amartya Sen (2003) propicia a partir de su postura frente a lo no vinculante del progreso económico medido en renta per cápita, y la calidad de vida valorada en morbilidad, en mortalidad o en esperanza de vida al nacer. La disyuntiva conceptual entre crecimiento y desarrollo económico, social y humano, indica su motivación por alejarse del modelo reduccionista que sitúa a la pobreza en términos de carencia financiera. La orientación de Sen (2003, p. 43) para evaluar el cambio social, medido en términos de la riqueza de la vida humana y esta, a su vez, expresada en la calidad de vida, es una propuesta que permite comprender la complejidad que implica la calidad de la vida humana.

El enfoque de capacidades es un marco teórico y conceptual amplio y general, en donde el eje central del análisis debe ser la información sobre las ventajas individuales evaluadas, desde un punto de vista de la oportunidad y no de un diseño específico de organización. Este enfoque se orienta principalmente a señalar la desigualdad de capacidades, en el análisis de las disparidades sociales (Sen, 2016). La construcción y el diseño de políticas públicas y sociales pueden estar influidos por la información que concentra el enfoque de capacidades, que puede contribuir, entre otras cosas, a un cambio en el énfasis de una política que se basa en el mejoramientos de los medios de vida a una que privilegia las oportunidades reales de vivir. 


\section{Método}

En esta sección se desarrolla el análisis de la información obtenida que permitirá profundizar en la comprensión de la construcción conceptual de los niños, las niñas en su ambiente natural de trabajo y que representa el modo en como esa realidad es conocida, interpretada y expresada. Esta metodología permitió hallar la interpretación de la experiencia del niño y la niña trabajadora en su entorno familiar, social y económico.

\section{Teoría fundamentada}

La teoría fundamentada propuesta por Glasser \& Strauss (1967) aporta de manera rigurosa un enfoque metodológico general de análisis comparativo, aplicado a una unidad social de cualquier tamaño, que induce a la predicción, la interpretación y otras aplicaciones relevantes, en el campo de la investigación social. La teoría fundamentada se presenta como un conjunto de proposiciones codificadas en un discurso teórico que se edifica a partir de categorías conceptuales y de sus propiedades. Esta estrategia de análisis comparativo produce una teoría propia del acontecimiento que se analiza, enfatizando en la teoría como proceso, la cual es útil para develar la realidad de la interacción social y la estructura contextual y no como un producto teórico perfeccionado (Glasser \& Strauss, 1967, p. 32).

El enfoque metodológico busca comprender la relación vinculante entre un sujeto (investigador) que desea conocer un objeto mediante «las acciones y significaciones de los participantes de la investigación» (Charmaz, 2013, p. 27). Según Strauss y Glasser (Strauss \& Corbin, 2002, 2016) la teoría fundamentada permite estructurar teorías emergentes a partir del análisis sistemático y contrastado de los datos cualitativos

Estos referentes teóricos se apoyan con similares ejercicios investigativos. En Páramo (2015) se reflexiona sobre el análisis de datos cualitativos en teoría fundamentada y en donde esta se valora como enfoque interpretativo, el cual se apoya en la sensibilidad del investigador con respecto a los significados que pueden ser evidentes como resultado del proceso de comparación de los contenidos, que emergen de las entrevistas y de la observación; estableciendo así diferencias y similitudes en los datos, lo que permite identificar categorías teóricas que ayudan a comprender el problema investigado (Bonilla \& López, 2016; Contreras \& Páramo, 2019; Estrada-Acuña et al., 2020).

Para el caso de esta investigación, se empleó el método de comparación constante. Además, se realizó el muestreo teórico para delinear las propiedades, semejanzas, diferencias 
e interrelaciones de las categorías que permitieron llegar al resultado teórico correspondiente (categorías emergentes). La técnica de análisis de los datos se cimentó en la codificación abierta, axial, lectura relacional, lectura selectiva y definición de categorías emergentes (Hernández, 2014).

Teniendo en cuenta que el proceso de análisis cualitativo lo anteceden unos resultados y hallazgos cuantitativos, se plantea un esquema de trabajo con un enfoque cualitativo con el mayor grado de sistematicidad y que permitiera generar un proceso de comparación y contrastación constante de datos, para luego proveer una construcción conceptual y teórica emergente sobre el problema social analizado. Igualmente, la elección de esta metodología se hace pertinente con el fin de prolongar el proceso de investigación a una fase posterior, dando continuidad al desarrollo de comparación de datos en otros escenarios aun no explorados. Una de las limitantes evidenciadas en el proceso es la no saturación parcial categorial, la cual permite abrir nuevas posibilidades de indagación de datos para ampliar el análisis.

\section{Recolección de los datos}

La investigación se sitúa en el municipio de Tello y en la ciudad de Neiva. El primero corresponde a un territorio cafetero y de interés por explorar las dinámicas de trabajo infantil en estos contextos agrícolas de notoria importancia, siendo la caficultura una de las actividades agrícolas más importantes en Colombia. La segunda es una de las ciudades capitales de Colombia con mayores dificultades en el mercado laboral, altas tasas de informalidad, desempleo y trabajo infantil.

Con la observación directa en campo se inició un proceso de participación pasivaactiva y, finalmente, completa. Una vez identificadas las características del entorno de los participantes, se realizó una segunda visita al lugar y se procedió aplicar el instrumento dispuesto para acopiar los datos. Se estableció para este fin la utilización de una entrevista semiestructurada y abierta, lo suficientemente flexible y cercana para establecer el diálogo entre el investigador y los niños y las niñas participantes. La constitución de las preguntas agrupa las categorías más relevantes (capital social, reconocimiento y capacidades), de tal modo que es el ritmo de la conversación con el niño o la niña lo que marca la posibilidad de ampliar las preguntas en el momento en que se realiza la entrevista, sin perder de vista los ejes categoriales que articulan el ejercicio investigativo.

Los estándares de calidad en el proceso de investigación cualitativa fueron orientados desde los lineamientos de Lincoln y Guba (1985) y en donde prevalecen los criterios de 
dependencia a partir de la pluralidad de la información, transferabilidad, en la posibilidad de continuar con una segunda fase de la investigación o de replicar este proceso investigativo en otros espacios territoriales, confirmabilidad en la revisión de los instrumentos por pares expertos y credibilidad en la recolección e interpretación de los datos.

\section{Unidad de trabajo}

En virtud de la búsqueda de profundidad sobre las dimensiones relacionadas con la construcción del capital social, el trabajo infantil en la dimensión del reconocimiento y las posibilidades de conciliar asistencia escolar con trabajo infantil, en una dimensión de las capacidades, se enfoca la selección de los participantes en este proceso, de manera intencional. Se decide entonces realizar las indagaciones en la zona rural del municipio de Tello, en donde se convocó de manera individual a cuatro niños y niñas. ${ }^{2}$ En la ciudad de Neiva se invitó a participar a un niño y una niña, quienes desarrollaban actividades de trabajo en un punto de la ciudad. Este es un tipo de muestra teórica, no probabilística u homogénea (Hernández-Sampieri et al., 2010), que se decide de manera intencional buscando que las categorías de la investigación estén en la muestra teórica elegida (Tójar, 2006), lo que va a permitir comprender los aspectos teóricos emergentes en torno a las dinámicas que motivan el trabajo infantil (tabla 1).

\section{Tabla 1}

Características de los participantes en la muestra teórica

\begin{tabular}{llcll}
\hline Participante & $\begin{array}{c}\text { Lugar o municipio } \\
\text { donde reside y trabaja }\end{array}$ & Edad & \multicolumn{1}{c}{ Tipo de trabajo } & Grado escolar \\
\hline Niño & Tello, vereda La reforma & 14 & $\begin{array}{l}\text { Agrícola. Recolección de café } \\
\text { dentro y fuera de la casa. }\end{array}$ & Octavo \\
\hline Niño & Tello, vereda La reforma & 12 & $\begin{array}{l}\text { Agrícola. Recolección de café } \\
\text { dentro y fuera de la casa. }\end{array}$ & Séptimo \\
\hline Niña & Tello, vereda La reforma & 11 & $\begin{array}{l}\text { Agrícola. Recolección de café en } \\
\text { casa. }\end{array}$ & Sexto \\
\hline Niña & Tello, vereda La reforma & 7 & $\begin{array}{l}\text { Agrícola. Recolección de café en } \\
\text { casa. }\end{array}$ & Segundo \\
\hline Niño & Neiva, barrio Galán & 9 & Trabajo ambulante & Segundo \\
\hline Niña & Neiva, barrio Galán & 14 & Trabajo ambulante & Séptimo \\
\hline
\end{tabular}

${ }^{2}$ Las madres y padres de familia autorizan la participación de cada uno de los niños y niñas en la investigación. El consentimiento informado, firmado por cada uno de ellos, así lo confirma. Los datos recopilados en el desarrollo de las entrevistas son de carácter anónimo y solo serán de interés investigativo. 


\section{Familias categoriales de modo apriorístico}

Las categorías se establecieron previamente enfocando la construcción y ajuste del instrumento (entrevista semiestructurada) con base en el diseño del marco teórico, de acuerdo con los objetivos que pretendía alcanzar la investigación. Su elección se basó en el desarrollo del marco teórico-conceptual. Asimismo, la construcción del instrumento se ajustó a esas mismas dimensiones categoriales con el fin de realizar un proceso de análisis de la información de datos cualitativos, partiendo de tres grandes familias categoriales, cada una con sus correspondientes subcategorías y estas, a su vez, definidas por sus descriptores de acuerdo con sus propiedades.

\section{Proceso de codificación y sistematización de los datos}

Definidas las familias categoriales, se obtiene un volumen importante de información que, en su conjunto, debe ser reducida para llegar a las conclusiones conceptuales y teóricas. Siguiendo la bitácora de manejo de datos cualitativos de Bonilla y Rodríguez (2000), se dividió la interpretación en dos niveles: el primero se relaciona con la etapa de codificación, categorización y reducción de datos, que permite elevar la exploración a un segundo nivel de análisis descriptivo de los resultados. La metodología en su conjunto permitió definir algunos patrones culturales, familiares y sociales sobre los factores que determinan para los niños y las niñas participantes la decisión de trabajar.

El proceso sistemático de codificación de los datos se realizó en cuatro etapas que permitieron depurar la información en un sentido inductivo. La primera fue la construcción del sistema categorial apriorístico que se fundamenta en tres familias categoriales: 1) El niño trabajador en una dimensión del reconocimiento y las capacidades; 2) la familia en la dimensión de la construcción del capital social; y 3 ) la educación y la escuela, nivel educativo en la dimensión de las capacidades y los funcionamientos. Una vez finalizada la lectura temática, se consolida la matriz de resultados con los códigos o palabras claves y su relación con las citas que corresponden a la respuesta que ofrece cada participante. La lectura temática y la depuración categorial que surge permite pasar a un tercer momento del análisis cuya fundamentación es la lectura relacional y la codificación axial; de esta forma se realizó un proceso de continua contrastación y comparación entre las respuestas de los participantes. Este nivel de codificación lleva a un análisis interpretativo a partir de la opinión de los participantes en torno a cada uno de los descriptores categoriales. El análisis que se presenta a continuación se relaciona con este primer resultado de contrastación entre las respuestas de los participantes para todas las subcategorías y los descriptores categoriales más relevantes. 


\section{Resultados}

\section{Interpretación y definición del campo categorial emergente}

El anterior proceso de codificación permitió establecer un análisis interpretativo con el fin de consolidar patrones homogéneos y relaciones entre las respuestas de los participantes. La reducción de las referencias se precisa aún más, orientado el proceso de análisis a la definición de las propiedades de las categorías. A partir de la codificación axial se propone un cuarto momento de codificación selectiva que define una serie de patrones emergentes que logran vincular de manera relacional los descriptores o palabras clave de cada subcategoría.

\section{Tabla 2}

Matriz de categorías emergentes de trabajo infantil rural y urbano para niños y niñas en los municipios de Tello y Neiva en el departamento del Huila

\begin{tabular}{|c|c|c|c|}
\hline Familias categoriales & Subcategorías & $\begin{array}{c}\text { Códigos/palabras } \\
\text { claves }\end{array}$ & Categorías emergentes \\
\hline \multirow{2}{*}{$\begin{array}{l}\text { El niño trabajador en una } \\
\text { dimensión del reconoci- } \\
\text { miento y las capacidades }\end{array}$} & Relación económica & $\begin{array}{l}\text { Remuneración } \\
\text { Tipo de trabajo } \\
\text { Entorno laboral }\end{array}$ & \multirow{2}{*}{$\begin{array}{l}\text {-El entorno del trabajo infantil } \\
\text { remunerado y no remunerado en } \\
\text { función del tipo de trabajo. } \\
\text {-El reconocimiento del trabajo en } \\
\text { función de la satisfacción o } \\
\text { insatisfacción }\end{array}$} \\
\hline & $\begin{array}{l}\text { Reconocimiento del } \\
\text { trabajo }\end{array}$ & $\begin{array}{l}\text { Economía familiar } \\
\text { Satisfacción/insatisfacción }\end{array}$ & \\
\hline $\begin{array}{l}\text { La familia en la dimensión } \\
\text { de la construcción del } \\
\text { capital social }\end{array}$ & Trabajo familiar & $\begin{array}{l}\text { Familia nuclear } \\
\text { Sustento familiar } \\
\text { Hogar como lugar de } \\
\text { trabajo }\end{array}$ & $\begin{array}{l}\text {-El trabajo infantil como aportan- } \\
\text { te en la consolidación del } \\
\text { sustento familiar en espacios de } \\
\text { trabajo dentro y fuera del hogar }\end{array}$ \\
\hline \multirow{3}{*}{$\begin{array}{l}\text { La educación y la } \\
\text { escuela } \\
\text { Nivel educativo en la } \\
\text { dimensión de las } \\
\text { capacidades y los } \\
\text { funcionamientos }\end{array}$} & Asistencia escolar & $\begin{array}{l}\text { Asistencia escolar y } \\
\text { desempeño educativo } \\
\text { Alimentación escolar }\end{array}$ & \multirow{3}{*}{$\begin{array}{l}\text {-La escolarización y los beneficios } \\
\text { - de la escuela como incentivo } \\
\text { para el desarrollo formativo de } \\
\text { NNJ } \\
\text {-Desempeño social en el entorno } \\
\text { escolar }\end{array}$} \\
\hline & & $\begin{array}{l}\text { Sociabilidad en la } \\
\text { escuela, pasatiempos, }\end{array}$ & \\
\hline & $\begin{array}{l}\text { Rol social en la } \\
\text { escuela }\end{array}$ & $\begin{array}{l}\text { qué le gusta hacer en el } \\
\text { colegio aparte de } \\
\text { estudiar } \\
\text { Anhelo futuro }\end{array}$ & \\
\hline
\end{tabular}

Entre los resultados producto de la reducción de los datos cualitativos a partir de la codificación se destacan cuatro aspectos emergentes que contienen las propiedades de los elementos categoriales, subcategoriales y descriptivos del conjunto de la información 
aportada por los niños y las niñas participantes. Tales categorías emergentes se definen para las tres familias categoriales en el siguiente orden:

1. El reconocimiento de los participantes del entorno de trabajo infantil cuando produce algún tipo de contraprestación monetaria y el trabajo que no produce este tipo de ganancia; en la exploración del trabajo infantil rural se vivencian los dos escenarios: «Siempre que trabajo fuera de mi casa recibo un pago por mi trabajo pero cuando trabajo en mi casa ahí si no me dan un salario. Yo me gano zo ooo al día». "Cuando yo trabajo en la finca de mi mamá no me pagan por que le estoy ayudando a ella; pero cuando voy a otra finca a mí me pagan por mi trabajo». Y en el caso del trabajo infantil urbano la práctica de la actividad es remunerada: «Todo el dinero que recibo se lo doy a mi mamá para la casa». En el mismo sentido del reconocimiento, la posibilidad de percibir en las vivencias propias de los niños y las niñas la satisfacción que produce en el trabajo infantil rural, su práctica: «iisi!!, me gusta trabajar porque yo puedo ayudar en el cafetal». «Sí..., toda la vida me ha gustado trabajar, porque así podemos comer». Y en el caso de la marcada insatisfacción que produce el desempeñar actividades de trabajo infantil en la ciudad: «No me gusta, pero tengo que hacerlo y si no hay para la comida».

2. La familia y el capital social; categoría emergente que asocia el trabajo infantil como un generador de ingresos y ayudas de otro tipo que aportan a la supervivencia del hogar; como palabra clave sustento familiar para el participante trabajador urbano sobresale la importancia de su contribución familiar por su trabajo en las calles: «Yo trabajo para poder ayudar a mi familia y poder comer nosotros. Todo el dinero es para mi familia». En el caso de los niños y niñas en la zona rural la connotación de aporte familiar necesario e indispensable no se hace tan notorio: «Pero cuando voy a otra finca a mí me pagan por mi trabajo. Trabajo a veces un día completo o a veces algún día del fin de semana. O sea, yo no trabajo fuera de mi casa todos los días. Yo este trabajo lo hago cuando llega la cosecha; por allá en mayo y en octubre». El hogar como lugar de trabajo es otra expresión descriptora clave que define un espacio de trabajo de niños y niñas rurales: «Solo trabajo en mi casa cogiendo café»; así como en los urbanos: «Ayudo a lavar los platos, a barrer y a trapear».

3. La categoría emergente relacionada con la educación como eje potenciador del desarrollo de competencias y habilidades sociales y formativas en los niños y las niñas. Los resultados se producen en torno a los incentivos que genera la asistencia escolar mediados por el suministro de alimentos e interacción social con pares en un entorno diferente al del hogar: «Cuando es el recreo yo juego futbol con mis 
compañeros del colegio; somos ocho compañeros en el curso. Mi mejor amigo se llama José Luis y me gusta mucho el fútbol; mi equipo favorito es el Nacional. Mi jugador favorito es James Rodríguez». «Jugar y comer la merienda. Juego con mis compañeros. Mi mejor amigo se llama Jaider Andrés. Mi deporte favorito es el fútbol y siempre lo juego en el recreo».

Se evidencian diferencias entre las prácticas de trabajo infantil rural y urbano en el sentido de cómo se asume desde la familia la participación de los niños y las niñas en las diferentes actividades. También se detectan diferencias con respecto a percepción que se tiene del trabajo como tal y del trabajo doméstico, considerado este último como la ayuda en el hogar.

\section{Discusión}

El desarrollo del trabajo de campo en el análisis cualitativo se orientó intencionalmente hacia participantes en condición de trabajo infantil rural y urbano para identificar y definir una construcción conceptual en torno a las razones y circunstancias por las cuales los niños trabajan.

El reconocimiento de los niños y niñas sobre lo que implica el trabajo remunerado y no remunerado y en cada uno de los entornos es relevante porque logra situar el objeto de estudio en el análisis de la información recopilada. Los niños y niñas rurales y urbanos perciben la diferencia entre el trabajo infantil generador de recursos monetarios y el trabajo infantil o ayuda (desarrollado en casa). Y en este sentido ellos y ellas se sitúan y se definen en el rol que les corresponda. En el caso de los y las participantes que residen en el campo, comprenden que las actividades que se realicen fuera de casa son trabajo remunerado: «Siempre que trabajo fuera de mi casa recibo un pago por mi trabajo, pero cuando trabajo en mi casa ahí si no me dan un pago». También se considera trabajo las actividades agrícolas realizadas en casa, pero no remuneradas: «Yo trabajo en mi casa desde los ocho años y lo primero que hice fue recoger café». En lo rural y lo urbano las actividades relacionadas con el cuidado del hogar y elaboración de alimentos se perciben como ayuda en casa. El escenario de la práctica del trabajo infantil rural está marcado por una intención familiar relacionada con aspectos asociados a la construcción de valores y normas que contribuyan a la educación y formación de los niños y niñas. Algunas investigaciones justifican el trabajo infantil con base en la construcción de valores en los 
infantes, relacionados con la autonomía, la responsabilidad, la disciplina y la habilidad de enfrentar dificultades (Padrón \& Román, 2013). Sin embargo, en el trabajo infantil urbano los participantes expresan que la práctica responde a una obligación por la búsqueda de un ingreso para solventar las necesidades básicas: «Tengo que hacerlo, y si no, no hay para la comida». Esta relación de pobreza como impulsor del trabajo infantil está ampliamente desarrollada en la literatura y, aunque no es el factor único que determine esta condición, siempre es importante tenerla presente en los análisis contextuales sobre el estudio problema social (Martínez, 2016; Simón et al., 2020; Ureña et al., 2009).

En la dimensión de la familia y el capital social destaca el hecho de los vínculos familiares que se construyen en función de las dinámicas propias de los hogares impulsados por fines comunes y en donde cada individuo asume un rol de responsabilidad. En el caso del trabajo infantil rural, la participación de los niños y niñas como trabajadores no se encuentra mediada estrictamente por la exigencia familiar que permita mejorar los ingresos, sino como un aporte al proceso de crianza en donde se acepta que niños y niñas asumen ciertas responsabilidades y roles de acuerdo a su edad y género. Este es un resultado coincidente con García (2015), quien concluye que niños y niñas que habitan en el campo construyen determinados saberes que forman parte de sus tradiciones y donde el trabajo infantil se entiende como un acto de participación y colaboración de los infantes al núcleo familiar.

Se observa una diferencia importante sobre lo que significa el trabajo para los niños en el campo o en lo rural. En este último, el trabajo es compartir con la familia, es ganar un rol social en el hogar, es el apoyo familiar. Las expresiones de los niños reflejan cierta complacencia. En cambio, el trabajo infantil en la ciudad indica el cansancio y el esfuerzo que demanda para ellos y ellas. Se percibe en los y las participantes una fuerte presión por la responsabilidad que sienten a su corta edad en función de ayudar en el hogar, a lograr suplir las necesidades básicas de alimentación y vivienda. Estas posturas de percepción opuestas sobre la práctica del trabajo infantil pueden, en parte, obedecer a la construcción familiar, que dadas las condiciones del entorno se constituyen y aceptan al interior del hogar y se refleja igualmente en lo que niños y niñas manifiestan sobre su actividad de trabajo desarrollada. Según Becerra y Cayeros (2017) los adultos construyen un discurso en torno al valor del trabajo, considerándolo como un elemento necesario para la formación y preparación para la vida futura de los niños y niñas. Esta investigación coincide en resaltar el interés que manifiestan los y las trabajadoras infantiles en identificar el trabajo como su interés principal, lo que puede definirse como su primera identidad. 
Frente a las posturas teóricas abordadas anteriormente, en las que se analizan las causas que originan el trabajo infantil y en las que se destacan aspectos relacionados con la pobreza, es evidente que la participación de los niños y las niñas - sobre todo en la ciudad - tiene mucho que ver con esta situación. En la zona rural aspectos como la provisión de alimentos y vivienda están resueltos en alguna medida y, en tal sentido, el trabajo infantil cobra otro significado relacionado, no solo con la práctica, sino con la necesidad de su existencia en el desarrollo social y de interacción familiar

Por último, se considera el aporte de esta investigación y sus resultados el vínculo en el análisis de elementos teóricos estructurados como el capital social, el reconocimiento situado y el enfoque de las capacidades, en clave para optimizar la comprensión sobre las causas del trabajo infantil en un análisis de contexto.

\section{Referencias}

Bar, T., \& Basu, K. (2009). Children, education, labor and land: In the long run and short run. Journal of the European Economic Association, 7(2/3), 487-497. https://doi.org/bt5wbm

Basu, K. (1998). The economics the child Labor. American Economics Review, 88(3), 412-422.

Becerra, S., \& Cayeros, L. (2017). Por el gusto de trabajar: construcciones, motivaciones y beneficios de la niñez en situación de trabajo en Tepic (Nayarit). Tla-Melaua, 11(43), 78-103. https://doi.org/10.32399/rtla.11.43.371

Berger, L., \& Luckman, T. (2003). La construcción social de la realidad. Amorrortu.

Bonilla, E., \& Rodríguez, P. (2000). La investigación en las ciencias sociales: más allá del dilema de los métodos. Ediciones Uniandes.

Bonilla, M., \& López, A. (2016). Ejemplificación del proceso metodológico de la teoría fundamentada. Cinta de Moebio, (57), 305-315. https://doi.org/gdvh4n

Bourdieu, P. (1986). The forms of capital. En J. G. Richardson (ed.), Handbook of theory and research for the sociology of education (pp. 240-268). Greenwood.

Carrión, V., Meneses, K., \& Cruz, E. (2021). Las preferencias de género en el trabajo infantil en Ecuador. Canadian Journal of Latin American and Caribbean Studies, 46(2), 180-195. https://doi.org/10.1080/08263663.2021.1882831

Coleman, J. (1988). Social capital in the creation of human capital. American Journal of Sociology, 94(95), 95-120.

Contreras, M., \& Páramo, D. (2019). La teoría fundamentada como metodología de construcción teórica. Pensamiento y Gestión, (7), 285-306. 
Charmaz, K. (2013). La teoría fundamentada en el siglo XXI: aplicaciones para promover estudios sobre la justicia social. En N. Denzin (Ed.), Estrategias de Investigación cualitativa (pp. 270-325). Gedisa.

Estrada-Acuña, R., Giraldo, C., \& Arzuaga, M. (2020). Aproximación al análisis de datos cualitativos en teoría fundamentada desde la perspectiva clásica. Revista Latinoamericana de Metodología de la Investigación Social, 20(10), 19-37.

Frasco-Zuker, L. (2016). El valor social del trabajo infantil: reflexiones a partir de una etnografía en Misiones [Ponencia]. X Jornadas de Sociología de la UNLP, 5 al 7 de diciembre del 2016, Ensenada, Argentina.

Fraser, N. (1997). ¿De la redistribución al reconocimiento? Dilemas en torno a la justicia en una época poscapitalista. En N. Fraser (Ed.), Justicia interrupta: reflexiones críticas desde la posición poscapitalista (pp. 17-54). Siglo del Hombres Editores.

García, V. (2015). El trabajo infantil rural: un puente para la construcción de saberes en la escuela rural. Infancias Imágenes, 15(1), 139-152. https://doi.org/gsxb

Glasser, G., \& Strauss, A. L. (1967). The discovery of grounded theory. Aladine Press.

Hernández-Sampieri, R., Fernández, C., \& Baptista, M. (2010). Metodología de la investigación. Mac Graw Hill.

Hernández, C. (2014). La investigación cualitativa a través de entrevistas: su análisis mediante teoría fundamentada. Cuestiones Pedagógicas, (23), 187-210.

Lefebvre, H. (1982). Lógica formal, lógica diléctica. Siglo XXI.

Lincoln, I., \& Guba, E. (1985). Naturalistic inquiry. Sage.

Martínez, A. (2016). Trabajo infantil en México: perfil sociodemográfico de los niños trabajadores de 5-11 años de edad. En D. Fernández, N. Tavira, \& R. E. Cavallero (Eds.), Trabajo global y desigualdades en el mercado laboral. (pp. 227-304). Clacso. https://doi.org/10.2307/j.ctvtxw2p2.14

Martínez, S. (2020). El proceso de agendación de la política pública para prevenir y erradicar el trabajo infantil (2011-2018) [Tesis de maestría]. Universidad Católica del Perú.

Organización Internacional del Trabajo. (2002). ¿Qué se entiende por trabajo infantil? https://www.ilo.org/ipec/facts/lang--es/index.htm

Organización Internacional del Trabajo. (2020). Covid-19 and child labour: A time of crisis, a time to act. Unicef.

Padrón, M., \& Román, P. (2013). Articularidades y complejidades del trabajo infantil: aspectos conceptuales y aproximación empírica a un fenómeno oculto con dimensiones diversas. Revista Facultad Ciencias Económicas, 21(1), 25-42. https://doi.org/gsxd Páramo, D. (2015). La teoría fundamentada. Pensamiento \& Gestión, (39), 1-7. 
Rausky, M. (2020). El estudio del trabajo infantil y los desafíos en su abordaje. Revista Colombiana de Sociología, 44(1), 317-340. https://doi.org/10.15446/rcs.v44n1.77594

Reséndez, M., Villanueva, A., Dimas de los Reyes, A., \& Dragustinovis, H. (2019). Trabajo infantil: una realidad social en el estado de Tamaulipas-México. Revista Ciencias Sociales, 25(4), 65-74. https://doi.org/10.31876/rcs.v25i4.30517

Salazar, M. (2000). El trabajo infantil en Colombia: tendencias y nuevas políticas. Nómadas, (12), 152-159.

Sen, A. (2003). Development as capability expansion. Oxford University Press.

Sen, A. (2016). La idea de la justicia. Taurus.

Simón, A., Guillen, H., \& Cueto, R. (2020). Creencias, actitudes y valores relacionados a la infancia y trabajo infantil en madres de Lima Metropolitana. Revista de Psicología, 38(1), 135-163. https://doi.org/10.1880o/psico.202001.006

Strauss, A., \& Corbin, J. (2002). Bases de la investigación cualitativa: técnicas y procedimientos para desarrollar la teoría fundamentada. Contus; Universidad de Antioquia.

Strauss, A., \& Corbin, J. (2016). Bases de la investigación cualitativa: técnicas y procedimientos para desarrollar la teoría fundamentada. Universidad de Antioquia.

Tójar, J. (2006). Investigación cualitativa: comprender y actuar. La Muralla.

Trujillo, J., \& Álvarez, N. (2011). Intercambio y mercado en el pensamiento crítico de Pierre Bourdieu. Apuntes del Cenes, 30(52), 9-36.

Unicef. (2006). Convención sobre los Derechos del Niño. Ginebra.

Ureña, A., Tovar, L. M., \& Castillo, M. (2009). Determinantes del trabajo infantil y la escolaridad: el caso del Valle del Cauca en Colombia. Revista Latinoamericana de Ciencias Sociales, Niñez y Juventud, 7 (2), 707-733.

Zelizer, V. (2002). Niño y comercio. Sociology, Gender Studies \& Cultural Studies, 9(4), $375-396$. 